\title{
"A Comparative Evaluation of Fracture Resistance of Endodontically Treated Teeth Obturated with AH Plus with GuttaPercha, Guttaflow with GuttaPercha and Resilon Epiphany- an Invitro Study."
}

\author{
Dr. M Kala Mds ${ }^{1}$, Dr. Shaktidar PR Mds ${ }^{2}$, \\ Dr. Allappanavarkeerti S Mds ${ }^{3}$, Dr. Gupthanagendra Vr Mds ${ }^{4}$ \\ ${ }_{1,2,3,4}$ (Department of Conservative Dentistry and Endodontics, GDCRI, Bangalore, India)
}

\begin{abstract}
Endodontically treated teeth have an increased predilection to fracture. The purpose of current study was to compare and evaluate the fracture resistance of root canal treated teeth obturated withdifferent materials. Seventy extracted single rooted human mandibular premolar teethwere decoronated and instrumented with hand files to a size 30 master apical file. The teeth were divided into three experimental groups of twenty teeth each and one control group of ten teeth.

Group 1: In this group the teeth were obturated with AH Plus and GuttaPercha.

Group 2: In this group the teeth were obturated with Gutta Flow and GuttaPercha.

Group 3: In this group the teeth were obturated with Resilon-Epiphany.

Group 4: Control group in which the teeth were unobturated.

All teeth were mounted vertically. Fracture strength testing was done using a universal testing machine (Lloyd $L R 50 K$, United Kingdom). The force required to fracture each tooth was recordedand analyzed. Teeth restored with AH Plus and guttapercha exhibited significantly higher resistance to fracture $(P<0.001)$. There was no statistically significant difference in fracture resistance between Gutta Flow and guttapercha, Resilon-Epiphany and control groups. it was concluded that the use of AH Plus and gutta-percha increased the fracture resistance of instrumented teeth compared with Resilon-Epiphany and Gutta Flow and gutta-percha.
\end{abstract}

Keywords: AH Plus, Fracture resistance, Gutta Flow, Guttapercha, Resilon-Epiphany.

\section{Introduction}

Endodontic therapy provides opportunities to maintain teeth in function and improve the health of the dentition. The long-term prognosis for endodontically treated teeth is greatly influenced by how well the coronal and apical seal are achieved. Root fracture, another reason for endodontic failure. Many in vivo studies have highlighted endodontic treatment as the major etiological factor for tooth fracture. ${ }^{1,2}$

The risk factors for fracture predilection in endodontically treated teeth are 1.Chemical factors: effects of endodontic irrigants and medicaments on dentine 2.Microbial factors: effects of bacteria-dentin interaction 3.Dentin factors: effects of tooth structural loss 4. Restorative factors: effects of post and core restorations 5 . Age factors: effects of age changes in dentin. ${ }^{2-5}$

Thus, it would be advantageous if the root canal obturation could reinforce the tooth and decrease the incidence of root fractures. The type of root canal sealer may affect root fracture resistance and the pattern of root fracture. ${ }^{6,7}$

Conventional root canal sealers do not bond strongly to dentin and gutta-percha, they do not behave as mechanically homogenous units with the root dentin. ${ }^{8}$ Interest in utilizing the classical monoblock concept for sealing and reinforcing the root canal space was rekindled with the advent of bondable root filling materials that are advocated as alternatives to conventional gutta-percha. It is thought that adhesion and mechanical interlocking between the material and root canal dentin will strengthen the remaining tooth structure, and thus reduce fracture risk. ${ }^{8,9}$

In 2004, a cold, flowable, self curingobturation material for root canals that combines GP and sealer into one injectable system. Effect of guttaflow on fracture resistance of the obturated teeth has not been studied previously. ${ }^{10}$

Though a number of sealers have been researched for their ability to reinforce tooth structure, studies on reinforcing ability of gutta flow in comparison to other sealers are lacking. This study was conducted to establish the fracture resistance of teeth subjected to compressive forces after obturation with GuttaPercha-AH Plus, GuttaPercha-Guttaflow and Resilon-Epiphany. 


\section{Materials And Methods}

Seventy mandibular premolar teeth that were extracted for orthodontic therapy were decoronated and their length was standardized to $13 \mathrm{~mm}$. The working length was established by placing no. $10 \mathrm{k}$-file into the root canal until it was visible at the apical foramen, and then subtracting $0.5 \mathrm{~mm}$ from that length. The teeth were instrumented to a size 30 master apical file. The step back technique was used to flare the canalfollowed by circumferential filing using $\mathrm{H}$-files. $10 \mathrm{ml}$ of $5.25 \%$ sodium hypochlorite was used as an irrigant in between successive files. After preparation the canal was irrigated with $10 \mathrm{ml} 5.25 \%$ sodium hypochlorite and $10 \mathrm{ml}$ of 17\% EDTA solution, each for 3 minutes to remove the smear layer. Final rinse was done with $10 \mathrm{ml}$ of distilled water. All canals were dried with paper points.

The teeth were divided into three experimental groups consisting of twenty teeth, one control group of ten teeth.

\section{Group I - GuttaPercha and AH Plus}

A standardized no. 30 size Guttapercha (Maillefer, Ballaiges, Switzerland) was selected as master cone, introduced into the root canal to full working length and was checked for tugback. AH Plus root canal sealer (DentsplyDetray, Konstanz, Germany) was mixed as per manufacturer's recommendations, equal volume units (1:1) of Paste A and Paste B was mixed on a mixing pad using a metal spatula to a homogeneous consistency. AH-Plus sealer was applied to the root canal wall using a finger spreader with a counter clockwise rotation. The apical part of the master cone was coated with AH-Plus sealer and introduced slowly into the root canal until the working length was reached. Lateral compaction was done using standardized finger spreaders (SybronEndo, Orange, CA) and accessory GuttaPercha cones (Maillefer, Ballaiges, Switzerland) coated with AH-Plus sealer was used.

\section{Group II - GuttaPercha and Gutta Flow.}

Guttaflow (ColteneWhaledent, Raiffeinsenstra, Langenau / Germany) was used in accordance with manufacturer's recommendations and teeth were obturated. A standardized 30 size gutta-percha (Maillefer, Ballaiges, Switzerland) was selected as master cone, introduced into the root canal to full working length and was checked for tugback. The GuttaFlow capsule was pressed to break the barrier between the powder and liquid and it was placed in a trituratorand allowed to vibrate for 30 seconds. The capsule plug was then removed and the disposable cannula attached to the capsule tip. The GuttaFlow plastic insertion tip was placed into the canal to a depth at which the tip no longer advanced. The GuttaFlow filling depth starting point was established $3 \mathrm{~mm}$ short of this length, the material was dispensed until it could be seen moving up the canal around the tip. The tip of the master point was coated with GuttaFlow paste and introduced into the root canal and was taken to the working length, slowly and gently back and forth, twice, to ensure complete wetting of the point and the canal wall. Then the master point was permanently placed in the canal. The canal was backfilled with GuttaFlow by placing the plastic insertion tip next to the master point to a depth at which the tip was neither forced nor bound the canal wall.

Group III - Resilon-Epiphany (Pentron, Wallingford, CT, USA)

The canals were conditioned using $10 \%$ polyacrylic acid, irrigated with sterile water and dried using paper points. A standardized 30 size resilon was selected as master cone, introduced into the root canal to full working length and was checked for tugback. The canals were filled with the primer using a pipette. The epiphany sealer was dispensed and mixed on a mixing pad and the teeth were obturated using resilon with the lateral compaction technique.

Group IV - This group consisted of 10 teeth, teeth in this group were left unobturated, serving as control.

\section{FIGURE 1: OBTURATION OF TEETH IN GROUP 1, GROUP 2 AND GROUP 3 RESPECTIVELY}
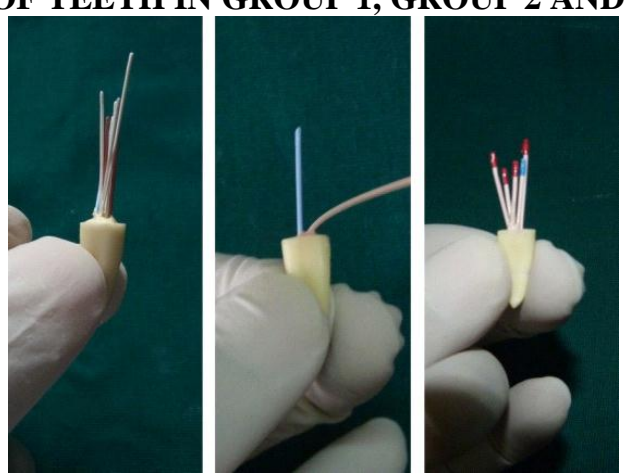

www.iosrjournals.org 
Excess material was seared off from the orifice and access cavity was sealed with Cavit G. The quality of root canal fillings was confirmed radiographically.

After one week, the specimens were prepared for mechanical testing. All teeth were mounted vertically with dental stone in PVC rings, exposing $8 \mathrm{~mm}$ of the tooth length. Fracture strength testing was done using a universal testing machine (Lloyd LR 50K, United Kingdom). A metal indenter of $5 \mathrm{~mm}$ diameter was fixed to the upper arm of the universal testing machine which was set to deliver an increasing load until fracture occurred. A cross head speed of $1 \mathrm{~mm} / \mathrm{min}$ was set and the load was applied vertically down to the long axis of the tooth. The force required to fracture each tooth was recorded in Newtons. The data thus obtained was evaluated statistically using One-Way ANOVA and Tukey's Post Hoc tests to determine the level of significance between different groups.

\section{Results}

The mean maximum load till fracture for each group in newtons is presented in Fig. 2

Higher mean Fracture resistance was recorded in AH-Plus with GuttaPercha followed by ResilonEpiphany group, GuttaFlow with GuttaPercha group and Control (Unobturated) group respectively. One way ANOVA test indicated that there was difference in Fracture Resistance between the groups was found to be statistically significant $(\mathrm{p}<0.001)$ (Table 1). Further, using Tukey's Post Hoc test, indicated the mean load till fracture for group 1 was statistically higher with respect to the other groups, whereas no significant difference was observed between the other pairs of groups ( $p>0.05$ ) (Table 2).

Figure 2: Comparison Of Mean Fracture Resistance Of Samples Among Study Groups

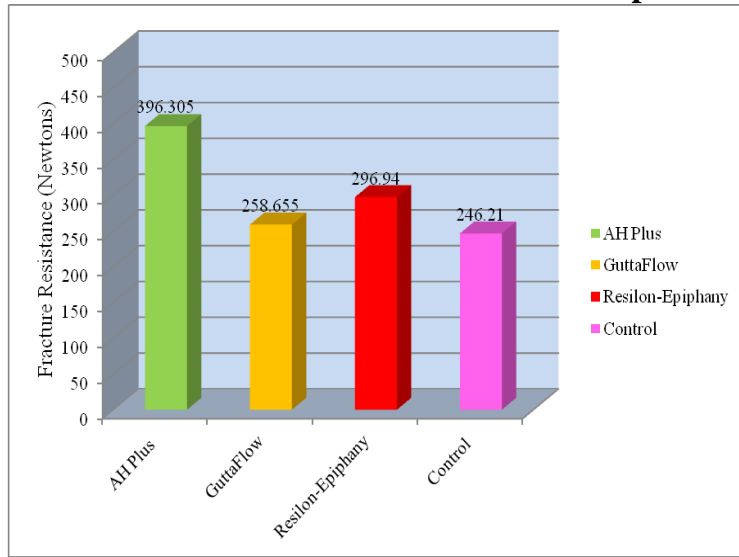

Table 1: Comparison Of Fracture Resistance (Newtons) Of Groups 1, 2, 3 And 4

\begin{tabular}{|c|c|c|c|c|c|c|}
\hline Group & N & Mean & SD & Median & 'F' value & \multirow{3}{*}{8.331} \\
\cline { 1 - 5 } Group 1 & 20 & 396.305 & 107.0565 & 386.300 & \multirow{2}{*}{$<0.001 *$} \\
\hline Group 2 & 20 & 258.655 & 95.5805 & 240.200 & \\
\hline Group 3 & 20 & 296.940 & 99.2092 & 268.650 & \\
\hline Group 4 & 10 & 246.210 & 87.6896 & 229.150 & & \\
\hline
\end{tabular}

*denotes significant difference, N- Sample size, SD- Standard Deviation

TABLE 2: INTERGROUP COMPARISON OF FRACTURE RESISTANCE

\begin{tabular}{|c|c|c|c|c|c|c|}
\hline \multirow[t]{2}{*}{ (I) Group } & \multirow[t]{2}{*}{ (J) Group } & \multirow{2}{*}{$\begin{array}{l}\text { Mean Difference } \\
(\mathrm{I}-\mathrm{J})\end{array}$} & \multirow[t]{2}{*}{ Standard Error } & \multirow[t]{2}{*}{$\mathrm{P}$ value } & \multicolumn{2}{|c|}{$95 \%$ Confidence Interval } \\
\hline & & & & & Lower Bound & Upper Bound \\
\hline \multirow[t]{3}{*}{1} & 2 & 137.6500 & 31.3231 & $.000^{*}$ & 55.091 & 220.209 \\
\hline & 3 & 99.3650 & 31.3231 & $.012^{*}$ & 16.806 & 181.924 \\
\hline & 4 & 150.0950 & 38.3628 & $.001^{*}$ & 48.982 & 251.208 \\
\hline \multirow[t]{2}{*}{2} & 3 & -38.2850 & 31.3231 & .615 & -120.844 & 44.272 \\
\hline & 4 & 12.4450 & 38.3628 & .988 & -88.688 & 113.558 \\
\hline 3 & 4 & 50.7300 & 38.3628 & .552 & -50.383 & 151.843 \\
\hline
\end{tabular}

$* \mathrm{p}<0.05$ Significant

\section{Discussion}

Endodontic procedure will remove sound tooth structure starting with the preparation of access cavity which may severely affect the strength and integrity of the tooth. In addition, intra canal preparation with instruments such as Gates glidden burs and various files will further remove tooth structure. Physical maneuvering during endodontic procedures in the form of access cavity, instrumentation of the pulp space and 
obturation may also lead to micro-cracks and fatigue that could further weaken the tooth structure. ${ }^{11}$ All these possibilities will render the endodontically treated teeth weaker than their vital counterpart.

Mandibular premolars were selected since they are appropriate for evaluation of the efficacy of materials to increase their fracture resistance. Their anatomy, function, crown size, and crown/root ratio may make them more prone to fracture than other posterior teeth.Moreover, considering their location in the dental arch, they are subjected to both compressive and shear forces.

There has been much controversy regarding the relative reinforcing power of the Resilon Epiphany system compared with AH-plus \& Gutta-percha. Although fracture resistance of teeth obturated with Resilon showed greater reinforcement compared with Gutta-percha, ${ }^{12,13}$ some authors have found a greater reinforcement power of Gutta-percha, ${ }^{14,15}$ other authors have found no significant difference between the two materials. ${ }^{9,16}$ In this present study, results show a lower resistance to fracture with the Resilon-Epiphany system compared with the AH-Plus and Gutta-percha group

AH plus sealer has greater adhesion to root dentin than Epiphany. This is likely due to the fact that, as an epoxy resin-based sealer, AH Plus has better flow properties because of its viscosity, its penetration into the microirregularities because of its creep capacity and long setting time, increases the mechanical interlocking between sealer and dentinal walls of the pulp space/ root canal. This fact, allied to the cohesion among sealer molecules, increases the resistance to removal and/or displacement from dentin, which can be translated as greater adhesion. ${ }^{14}$

A study showed epiphany bonded poorly to the dentinal wall, hence they are unlikely to reinforce root strength in clinical situations. ${ }^{17,18}$ Another aspect that could interfere in the polymerization reaction of the root canal sealer is its incomplete photoactivation in the whole extension of the specimen, which results in the presence of unreacted residual monomers in the deepest portion of the specimen. ${ }^{14}$

In root canals, C-factors can be over 1000. Any polymerizing endodontic sealer will be subjected to large polymerization stresses during their setting that may cause debonding and gap formation along the periphery of the root filling. The extremely high C-factor in root canals has been cited as a possibility for not achieving perfect seals in Resilon-filled root canals. ${ }^{8}$

For Resilon, or any endodontic sealer to reinforce roots, it not only must bond to the root canal walls, but it should also have a modulus of elasticity approaching that of dentin, which is about $17 \mathrm{GPa}$. The flexural modulus parameter defines the flexibility of a sample and higher values indicate more stiffness, whereas lower values indicate more flexibility. Despite the fact that Resilon showed a statistically higher flexural modulus than Gutta-percha, Resilon and Gutta-percha are not expected to reinforce roots per se because both have a very low modulus of elasticity (Resilon $=200.28 \pm 17.68 \mathrm{Mpa}$ and Gutta-percha $=292.65 \pm 38.43$ ). This confirms that the stiffness of Resilon and Gutta-percha is too low to reinforce roots. Furthermore, the data from Gutta-percha in this study demonstrated a statistically higher flexural modulus than Resilon ( $\mathrm{p}=0.000)$, which was higher than the modulus of elasticity previously reported for Gutta-percha $(77 \mathrm{Mpa}) .{ }^{19}$

The compressive force used in this study fundamentally differed in nature from masticatory forces. A compressive force with cross head speed of $1 \mathrm{~mm} / \mathrm{min}$ was applied using a metal indenter of $5 \mathrm{~mm}$ diameter. While natural forces change rapidly in type, magnitude, and direction, this force was a static compressive force that gradually increased until breakage occurred. In addition, thermocycling and the periodontal ligament were not simulated in this study. Therefore, clinical studies are necessary to evaluate these findings further.

\section{Conclusion}

Under the limitations of the present study it can be concluded that the fracture resistance of teeth obturated with AH Plus and GuttaPercha were significantly superior to teeth obturated with Guttaflow and GuttaPercha, and Resilon-Epiphany.

\section{References}

[1] Hulsmann M, Peters OA, Dummer PM. Mechanical preparation of root canals: shaping goals, techniques and means. Endodontic Topics, 10, 2005, 30-76.

[2] Kishen A. Mechanisms and risk factors for fracture predilection in endodontically treated teeth. Endodontic Topics, 13, 2006, 5783.

[3] Sedgley CM, Messer HH. Are endodontically treated teeth more brittle? Journal of Endodontics, 1992;18(7):332-335.

[4] Peroz I, Blankenstein F, Lange KP, Naumann M. Restoring endodontically treated teeth with posts and cores- a review. Quintessence int, 36(9), 2005, 737-746.

[5] Ricks-Williamson LJ, Fotos PG, Goel VK, Spivey JD, Rivera EM, Khera SC. A three dimensional finite element analysis of an endodontically prepared maxillary central incisor.Journal of Endodontics, 21(7), 1995, 362-367.

[6] Chadha R, Taneja S, Kumar M, Gupta S. An in vitro comparative evaluation of depth of tubular penetration of three resin based root canal sealers. Journal of Conservative Dentistry, 15(1), 2012, 18-21.

[7] Lertchirakarn V, Timyam A, Messer HH.Effects of Root Canal Sealers on Vertical Root Fracture Resistance of Endodontically Treated Teeth.Journal of Endodontics, 28, 2002, 217-219.

[8] Tay FR, Pashley DH. Monoblocks in Root Canals: A Hypothetical or a Tangible Goal. Journal of Endodontics, 33(4), 2007,391 398. 
[9] Wadhwani KK, Gurung S. Evaluation of root canal sealers on the fracture resistance of root canal treated teeth - An in vitro study. Endodontology, 22, 2010, 51-56.

[10] Kandaswamy D, Venkateshbabu N, Krishna RG, Hannah R, Arathi G, Roohi R. Comparison of laterally condensed, vertically compacted thermoplasticized, cold free-flow GP obturations- A volumetric analysis using spiral CT. Journal of Conservative Dentistry, 12(4), 2009, 145-149.

[11] Upadhyay V, Upadhyay M, Panday RK, Chturvedi TP, Bajpai U. A SEM evaluation of dentinal adaptation of root canal obturation with guttaflow and conventional obturating material.IJDR, 22(6), 2011, 881-884.

[12] Baba SM, Grover SI, Tyagi V. Fracture resistance of teeth obturated with Guttapercha and Resilon: An in vitro study. Journal of Conservative Dentistry , 13, 2010, 61-64.

[13] Sakri MR, Shetty D, Hindhuja D, Byakod P, Nageswar R. RESILON - "ENDOBONDICS". Pravara Med Rev 2010;5(1):4-9.

[14] Nunes VH, Silva RG, Alfredo E, Sousa-Neto MD, Silva-Sousa YT.Adhesion of Epiphany and AH Plus Sealers to Human Root Dentin Treated with Different Solutions. Brazilian Dental Journal, 19(1), 2008, 46-50.

[15] Gesi A, Raffaelli O, Goracci C, et al..Interfacial Strength of Resilon and Gutta-Percha to Intraradicular Dentin.Journal of Endodontics, 31(11), 2005, 809-813.

[16] Javaheri M, Bahmani-Zanjani L, Rakhshan V, Foroozia M. Vertical fracture resistance of endodontically treated teeth restored with four sets of obturation and filling materials. Journal of Dental Sciences, 7, 2012, 130-136.

[17] Bouillaguet S, Bertossa B, Krejci I, Wataha JC, Tay FR, Pashley DH. Alternative Adhesive Strategies to Optimize Bonding to Radicular Dentin.Journal of Endodontics, 33(10), 2007, 1227-30.

[18] Kim YK, Grandini S, Ames JM, et al. Critical Review on Methacrylate Resin-based Root Canal Sealers. Journal of Endodontics, 10, 2007, 1-17.

[19] Grande NM, Plotino G, Lavorgna L, et al. Influence of Different Root Canal-Filling Materials on the Mechanical Properties of Root Canal Dentin.Journal of Endodontics, 33(7), 2007, 859-63. 